\title{
DEVELOPMENT AND MODELING OF ROBOTIC MANUFACTURING CELL FOR EXPERIMENTAL PURPOSES
}

\author{
IVAN, A[ndrei] - M[ario]; NICOLESCU, A[drian] F[lorin]; DOBRESCU, T[iberiu] G[abriel] \& STRAJESCU, \\ E[ugen] R[adu]
}

\begin{abstract}
The article presents the research performed by the authors in the field of robotic machining with self-driven tools, focusing on low power deburring operations performed by six degrees of freedom articulated arm robots. The works include layout development, modeling and simulation of a flexible manufacturing cell for machining operations. The research presented in this article is focused on two main goals: first, to show and evaluate the influence of trajectory target points coordinates (determined by the workpiece installation position) on machining parameters and force/torque values in each robot's joint; and second, to elaborate the mathematical model of the robot arm in order to use it in future research as an intermediate stage of comparison between the theoretical model used in virtual environment simulations and the real robotic arm structure and behaviour in experimental operations.
\end{abstract}

Key words: robotic manufacturing cell, machining, kinematic model, dynamic model, workpiece positioning

\section{INTRODUCTION}

Continuing previously presented papers, the present work highlights third level approach performed by authors in the field of specific research for robotic machining. First level approach has been related to performing a documentation study to identify state of the art in robotic systems architectures, specific end-effectors and robotic machining processes for complex part manufacturing. Second level approach has been related to virtual prototyping and simulation of optimized flexible manufacturing cells specially dedicated for robotic machining using self-driven tools, including specific devices for supplementary part orientation. Present work is directly related to a dedicated approach on specific modeling techniques related to robotic manufacturing systems using self-driven tools. The scope of the research was to develop the concept, layout and modeling of a robotic manufacturing cell for experimental applications in the field of self-driven tool machining, focusing on deburring low power operations (machining power up to $800 \mathrm{~W}$ ). Because the analysis is centered on the robot's mechanical structure, the workpiece mounting table stiffness and other characteristics have not been taken into consideration. Further, the analysis was simplified by assuming that the tool remains in vertical position throughout the machining process.

\section{THE LAYOUT PRINCIPLE OF THE ROBOTIC MANUFACTURING CELL}

The developed manufacturing cell presented in this paper corresponds to the first robotic machining cell layout principle (as classified in Nicolescu \& Ivan, 2009), being dedicated for low-power machining operations.

The structure of the manufacturing cell includes a Kawasaki FS10E 6-axis articulated arm industrial robot with 10 kg payload, equipped with an ATI RC 340E radially compliant deburring end-effector featuring $40000 \mathrm{rpm}$ spindle and $340 \mathrm{~W}$ machining power connected through an automatic ATI QC 041ET tool changer. A Kistler 9257B dynamometer is mounted between the workpiece and the table, with the purpose of measuring the machining forces and torques (Nicolescu, 2005). The experimental part used was a 6082 aluminium alloy plate with 200x160 mm dimensions. The overall layout of the robotic machining cell is illustrated in Fig. 1. The purpose of the experimental setup is to determine the maximum values of the machining parameters with respect to processed material characteristics. The structural layout of the manufacturing cell was elaborated, optimized and validated through modeling and simulation in the virtual environment offered by the $\mathrm{ABB}$ RobotStudio software. The main goal of the simulation was to determine the required height of the robot pedestal and the optimum position of the workpiece with respect to robot's base coordinate system, as well as to validate the cell's workspace distribution and robot arm kinematic model.

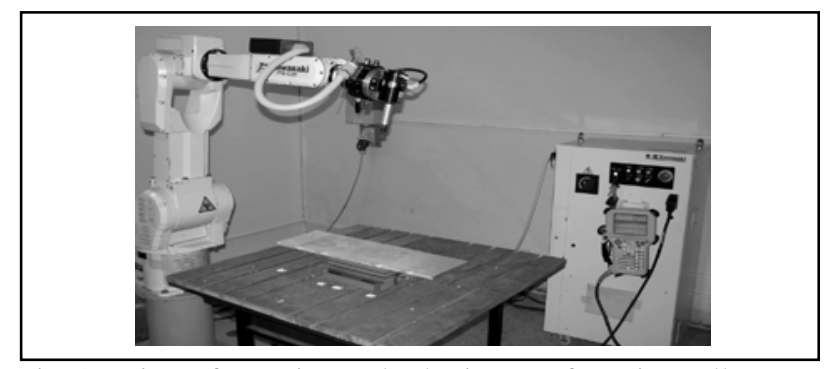

Fig. 1. View of experimental robotic manufacturing cell

\section{MANUFACTURING CELL MODELING AND SIMULATION IN VIRTUAL ENVIRONMENT}

In order to validate and optimize the structural distribution of the general system components, a virtual model based on the real manufacturing cell layout principle was created. Subsequently, the virtual model was configured for use with the ABB RobotStudio programming and simulation software package, as shown in Fig. 2.

The modeling, optimization and validation process is based on the following concepts:

- The robot's base coordinate system (noted $\mathrm{X}_{0} \mathrm{Y}_{0} \mathrm{Z}_{0}$ ) was considered to be the reference coordinate system of the application. The part's position is thus expressed with respect to this coordinate system and is described by considering the geometrical centre of the part as the origin of the workpiece coordinate system.

- The main issue with robotic machining (using articulated arm robots) is the relatively low stiffness of the serial structure of the robot arms, which determines errorgenerating events during the process, such as chatter and joints elastic displacement (Pan \& Zhang, 2007). Considering these aspects, it is desirable to keep the machining forces and torques as low as possible, knowing 
that the torques acting on the robot's joints also depend on the position of the tool-part contact point with respect to the robot's base.

- The machining is made by programming the TCP to follow a certain path, interpolated from a set of target points specified by the programmer (Mitsi et al., 2004). By estimating the machining force and torque values in each of the target points, the results can be used for comparison between the different configurations and workpiece positions in order to determine the workpiece position which generates the global lowest forces and torques in each joint taking into account all trajectory target points.

- An important factor in programming articulated arm robots is the occurrence of singularity situations. This factor depends mainly on path starting robotic arm configuration, but is also influenced by the part's position with respect to the robot. While near singularity configurations robot's mobility is greatly reduced (small velocities in the operational space will generate very large velocities in joint space), Yoshikawa's manipulability model was used to determine if the robot arm is close to a singularity situation, defined as:

$$
w(\vec{q})=|\operatorname{det}(J(\vec{q}))|
$$$$
\rightarrow
$$

where $\vec{q}$ is the joint variables vector and $J$ the Jacobian matrix (Yoshikawa, 1985). The expressed manipulability is positive and equal to zero at a singular configuration, so that it is desired to have high values for $w(\vec{q})$, which indicates high mobility of the robot's arm.

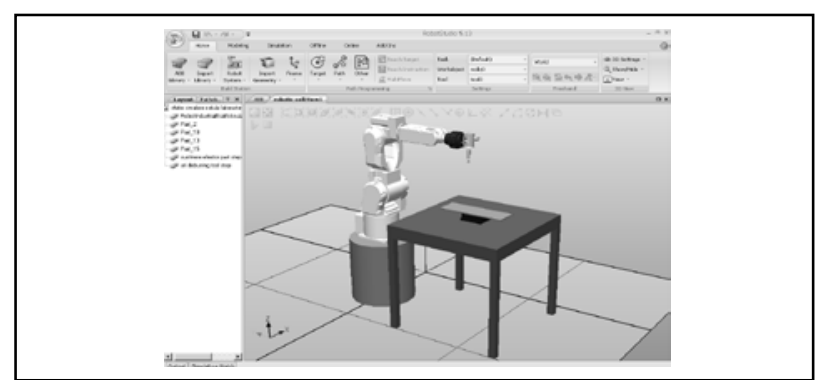

Fig. 2.Virtual model of the developed robotic manufacturing cell

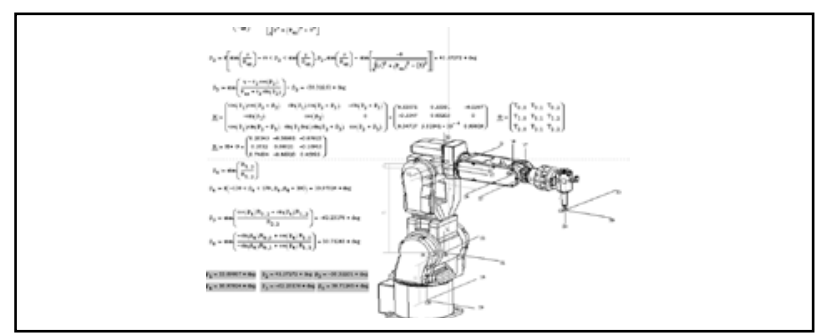

Fig. 3.Inverse kinematics of the Kawasaki FS10E robot arm computed in Mathcad

Following the general principles exposed above, the simulation and cell workspace analysis were performed, together with Denavit-Hartenberg parameters identification, kinematic modeling of the system and Jacobian matrix formulation, followed by speed and trajectory control calculations. Further, the dynamic model of the robot arm was derived using the Lagrange-Euler method (Spong, 2006). These mathematical models were computed using Mathcad software (as shown in Fig. 3) and the results were used for joint torques evaluation for trajectory target points.

The optimal point for the machining process (the point which offers the best compromise between machining forces / torques values, robot arm mobility and singularity situations occurrence) was determined to be at a distance of $660 \mathrm{~mm}$ from the robot's base joint (measured in the direction of the $\mathrm{Y}_{0}$ axis). The mathematical analysis showed that, above the mentioned value, the machining torques increase so that, above $1000 \mathrm{~mm}$ distance from the robot's base joint, regenerative chatter and significant profile errors occur. Also, below the optimal value, the manipulability of the robotic arm decreases to the point that the trajectory could not be followed without intermediate reconfigurations of the robotic arm.

\section{CONCLUSION}

The cell presented in this paper was modeled going through all specific development phases, from component distribution, workspace validation and system integration, to application programming and simulation, using for each step specific computer aided methods for modeling and calculations. The works performed revolved around two major goals:

- To determine the robot workspace domain which offers the best compromise between machining forces/torques values in each joint of the robot's mechanical structure, robot arm mobility and singularity situations occurrence, and to evaluate the influence of the target points coordinates (directly determined by the workpiece mounting position and orientation, expressed relatively to robot's base frame) on machining process parameters.

- To completely model the mechanical structure of the robotic system (kinematics, speed and trajectory control, dynamic behavior) in order to offer terms of comparison between the theoretical model used in virtual environment simulations and the real robotic arm structure and behavior in experimental operations.

Future research will be focused on performing experimental machining operations on various types of materials in order to determine the maximum values for the machining parameters with respect to material hardness. Another goal will be to find and model the relation between robotic arm stiffness and generated surface errors, also considering the forces at the toolworkpiece contact area. Also, a comparative analysis between virtual modeling and simulation outputs and the results obtained from experimental operations will be performed in order to improve offline programming approaches for industrial robots and provide methods for offline program optimization.

\section{REFERENCES}

Mitsi, S.; Bouzakis, K.-D.; Mansour, G.; Sagris D. \& Maliaris G. (2004). Off-line programming of an industrial robot for manufacturing, The International Journal of Advanced Manufacturing Technology, Vol. 26, No. 3, (August 2005), pp. 262-267, ISSN 0268-3768

Nicolescu, A. (2005). Industrial Robots, EDP Publishing House, ISBN 973-30-1244-0, Bucharest, Romania

Nicolescu, A. \& Ivan, A. (2009). Actual development status in robotic machining - a survey, Proceedings of the 18th International Workshop on Robotics in Alpe-Adria-Danube Region, May 25-27, Brasov, Romania, ISBN 978-606-521315-9, Printech, 2009

Pan, Z. \& Zhang, H. (2007). Analysis and suppression of chatter in robotic machining process, International Conference on Control, Automation and Systems, Oct. 1720, Seoul, Korea

Yoshikawa, T. (1985). Manipulability of robotic mechanisms, International Journal of Robotic Research, , Vol. 4, No. 2, (June 1985), pp. 3-9, ISSN 02783649

Spong, M.; Hutchinson, S. \& Vidyasagar, M. (2006). Robot Modeling and Control, John Wiley \& Sons, Inc., ISBN 978-0-471-64990-8, Hoboken, NJ, USA 